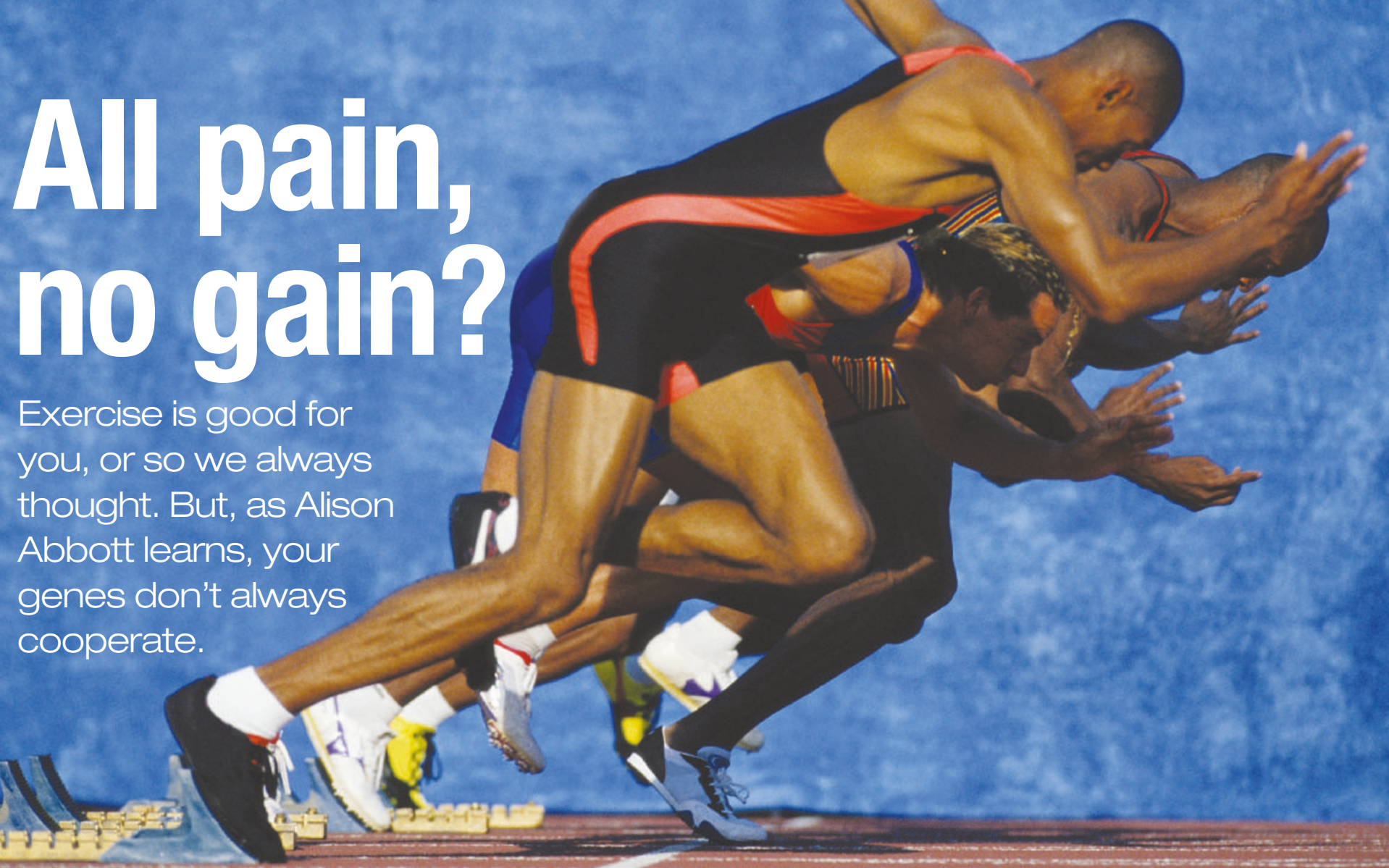

W hen Claude Bouchard set out to see whether genes play a role in physical fitness, he assumed, like most people, that exercise training makes everyone fitter. Although he expected genes to modulate some individual responses to diet and exercise, he also anticipated that regular workouts would improve fitness indicators such as lung efficiency and blood cholesterol for everybody.

Some 20 years later, it has become clear from the work of Bouchard and others that this is not the case. Looking at certain measures of fitness, some people actually fare worse after exercise, whereas others show little or no improvement.

But this isn't vindication for couch potatoes. Everyone's health improves in some way or other from exercise, but just how it improves is largely dependent on genes. Now, the growing field of fitness genetics is attempting to tease those genetic components apart, and the studies are generating fresh insights into the benefits of exercise as well as unexpected pay-offs for medicine.

Bouchard's attempts to track fitness genes began in the mid-1980s at Laval University in Quebec, Canada. He and his colleagues focused on the maximum amount of oxygen absorbed by the body from a lungful of air a standard measure of aerobic fitness, usually abbreviated as $V_{\mathrm{O}_{2} \max }$. They found that most people can get more oxygen out of each breath after training but that a minority were no better off, regardless of how efficient their lungs were at the start. Because the variation was much less extreme within pairs of identical twins, Bouchard concluded that the effect was largely dictated by genes ${ }^{1}$.

That initial study was fairly small, so Bouchard extended the work in 1992 by helping to set up a multicentre research effort called the HERITAGE Family Study, which is still running today. Now based - together with Bouchard - at the Pennington Biomedical Research Center in Baton Rouge, Louisiana, the study's main data set comes

"Our work on athletes is feeding back into the clinic. How efficiently we use oxygen is decisive when we are desperately sick."

- Hugh Montgomery

from some 740 sedentary adults who were subjected to an intense exercise regime in the lab. The researchers monitored changes in the participants' blood pressure, heart rate, blood chemistry and $V_{\mathrm{O}_{2} \max }$ over 20 weeks.

\section{Survival of the fittest}

The study's main aim was to determine how exercise reduces risk factors for cardiovascular disease and diabetes, but Bouchard and researchers at the four other collaborating institutions also took blood samples for genetic analysis. "We were trying to find as many genes as possible that influence fitness and performance," Bouchard says.

The resulting reams of data and frozen blood samples are still being analysed, but the results so far confirm Bouchard's earlier stud- ies. The average increase in $V_{\mathrm{O}_{2} \max }$ after the training programme was $19 \%$. But $5 \%$ of the subjects had virtually no change, and another $5 \%$ had improved by more than twice the average amount. Similarly, most people had lower exercising heart rates and blood pressure after the training programme - an indication of improved fitness but the extent of the reduction was extremely variable. In a few people there was even a small rise in these numbers ${ }^{2}$.

Much of this variability seems to be attributable to genes. The researchers found more variation between than within families, suggesting at least a portion of a person's ability to benefit from exercise is inherited. "We concluded that just about half of the difference in trainability was heritable," says Tuomo Rankinen, the study's project manager.

It is unclear to what extent fitness parameters such as $V_{\mathrm{O}_{2} \max }$ are indicative of longterm health prospects, but even presumed health indicators such as cholesterol, a factor in heart disease, did not follow the expected pattern of more exercise is better. Conventional wisdom has it that regular exercise reduces the risk of heart disease by raising blood levels of high-density lipoprotein (HDL) cholesterol, a complex that helps prevent cholesterol from forming fatty deposits on blood-vessel walls. This is considered one 
